\title{
Independent estimates of energies of steep-spectrum radio sources
}

\author{
Alla Miroshnichenko \\ Institute of Radio Astronomy of NAS of Ukraine \\ UA-61002, Kharkov, Ukraine \\ email: mir@rian.kharkov.ua
}

\begin{abstract}
We examine properties of galaxies and quasars with steep low-frequency spectra from the UTR-2 catalogue. The number of the objects have the non-thermal X-ray emission due to the inverse Compton scattering of radio photons of the microwave background by relativistic electrons. So, it is possible to estimate the magnetic field strength and the ratio of energies of the magnetic field and relativistic particles, independently. As we have received the determined values of magnetic field strength are near to one order less than those at the well-known energy equipartition condition. We conclude from the obtained energy ratio that the energy of relativistic particles prevails over the energy of magnetic field in the galaxies and quasars with steep radio spectra.
\end{abstract}

Keywords. Steep radio spectrum, magnetic field strength, relativistic particles, energy

\section{Introduction}

As it follows from the UTR-2 catalogue of extragalactic sources at the decametre band ( Braude et al. 1978, Braude et al. 1979, Braude et al. 1981a, Braude et al. 1981b, Braude et al. 2003 ) near 30 percent of sources have steep radio spectra (the spectral index is $\alpha \geqslant 1$ ). According to conclusion by Kardashev (1962), the critical frequency of the synchrotron emission of radio source can displace to value less than $10 \mathrm{MHz}$ when the electron acceleration is not continuous.So, the radio spectrum will be steep. Note, that galaxies and quasars with steep radio spectrum posses the very extent radio structure $(\sim \mathrm{Mpc})$ and the great luminosity $\left(\sim 10^{28} \mathrm{~W} /(\mathrm{Hz}\right.$ ster $)$ at the frequency $\left.25 \mathrm{MHz}\right)$ (Miroshnichenko 2012 ).

Sources from the UTR-2 catalogue display two types of steep radio spectra: the linear (S) and the break spectra (C+) (Fig. 1).

A number of galaxies and quasars with steep radio spectrum are the sources of nonthermal X-ray radiation. In this case we have the possibility of alternative determination of the magnetic field strength in the sources. It is necessary for the estimate of the magnetic field energy and the energy of the relativistic particles. For this aim we consider galaxies and quasars with steep radio spectrum in the UTR-2 catalogue.

\section{Samples of sources with steep spectrum from the Grakovo (UTR-2) catalogue}

The examined samples have been compiled from sources with steep spectrum of two types - with linear steep spectrum $(\mathrm{S})$ and low-frequency steepness after a break $(\mathrm{C}+)$. Within the declination ranges $\delta$ from -13 to +20 degrees and from +30 to +40 degrees the steep-spectrum sources have been selected with flux density more than $10 \mathrm{Jy}$ at $25 \mathrm{MHz}$. 

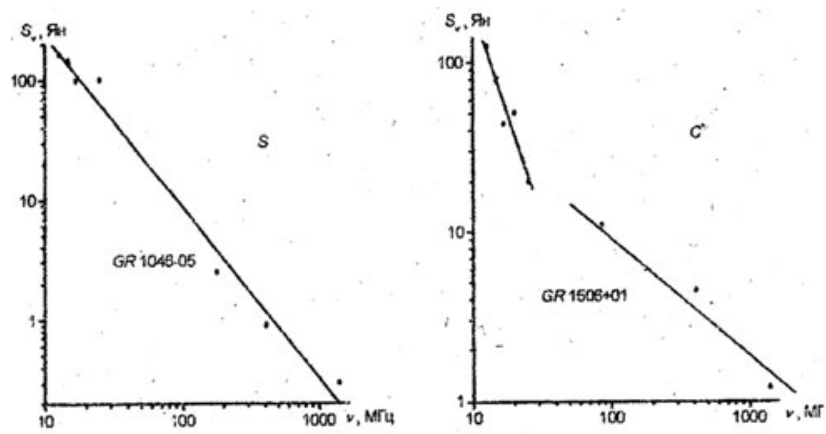

Figure 1. Examples of steep spectra from the UTR-2 catalogue of extragalactic radio sources.

The sample of objects with linear steep spectrum (type S) contains 78 galaxies (from which 11 are the X-ray sources), and 55 quasars (from which 23 are the X-ray sources). The sample of objects with steepness spectrum (type $\mathrm{C}+$ ) contains 52 galaxies (from which 10 are the X-ray sources), and 36 quasars (from which 15 are the X-ray sources). The optical and high-frequency data for examined sources have been got from the NED database. The redshift range of objects is enough vast and forms $z=0.017 \ldots 3.570$.

The mean spectral indices and mean value of redshifts are: (sample (S)) for galaxies $\langle\alpha\rangle=1.21 \pm 0.002,\langle z\rangle=0.697 \pm 0.105$; for quasars $\langle\alpha\rangle=1.24 \pm 0.003,\langle z\rangle=1.029 \pm$ 0.07; (sample $(\mathrm{C}+)$ ) for galaxies $\langle\alpha\rangle=2.01 \pm 0.08$ (at the decametre band), $\langle z\rangle=0.308 \pm 0.076$; for quasars $\langle\alpha\rangle=2.25 \pm 0.14$ (at the decametre band), $<z>=0.950 \pm 0.092$.

\section{Independent estimation of the magnetic field strength and energies of steep-spectrum sources}

The independent estimation of the magnetic field strength $\left(B_{I C}\right)$ of sources is possible at the assumption that X-ray emission of considered sources may be due to the inverse Compton scattering (IC) of radio photons of the microwave background by relativistic electrons.

To obtain $B_{I C}$ by the flux density of X-ray emission and the flux density of radio emission of source, we transform the relation by Harris\& Grindley (1979) :

$$
B_{I C}=\left[\left(5.05 \cdot 10^{4}\right)^{\alpha} \cdot 1.15 \cdot 10^{-16} \cdot(1+z)^{\alpha+3} \cdot S_{r} \cdot \nu_{r}^{\alpha} \cdot S_{X}^{-1} \cdot \nu_{X}^{-\alpha}\right]^{\frac{1}{\alpha+1}} \text { (Gauss) }
$$

where all values are in CGS system, $\nu_{x}=2.42 \cdot 10^{17} \mathrm{~Hz}, \nu_{r}=2.5 \cdot 10^{7} \mathrm{~Hz}$. Besides, for comparison, we have determined the corresponding values of magnetic field strength of examined sources at the energy equipartition condition $\left(B_{e q}\right)$. As a result of calculations, the estimates of the magnetic field strengths are:

(sample $(\mathrm{S}))$ for galaxies $\left\langle B_{I C}\right\rangle=0.98( \pm 0.42) \cdot 10^{-6}$ Gauss, $\left\langle B_{e q}\right\rangle=4.11( \pm 0.24)$. $10^{-5}$ Gauss; for quasars $\left\langle B_{I C}\right\rangle=2.23( \pm 0.41) \cdot 10^{-6}$ Gauss, $\left\langle B_{e q}\right\rangle=3.1( \pm 0.10) \cdot 10^{-5}$ Gauss;

(sample $(\mathrm{C}+))$ for galaxies $<B_{I C}=0.88( \pm 0.21) \cdot 10^{-6}$ Gauss, $<B_{e q}>=8.14( \pm 0.10)$. $10^{-6}$ Gauss; for quasars $\left\langle B_{I C}>=1.69( \pm 0.29) \cdot 10^{-6}\right.$ Gauss, $\left\langle B_{e q}\right\rangle=6.15( \pm 0.30) \cdot 10^{-6}$ Gauss.

So, the derived values $B_{I C}$ are, in average, to by one order less than the values $B_{e q}$ for the steep-spectrum galaxies and quasars. 
We may estimate the energy of relativistic electrons of examined sources from the expression by Lang(1974) using in our case the values of $B_{I C}$ and radio luminosity $L$ at the low-frequency range from $\nu_{1}=10 \mathrm{MHz}$ to $\nu_{2}=100 \mathrm{MHz}$ :

$$
W_{e}=\frac{10^{12} L}{B_{I C}^{3 / 2}} \frac{\nu_{2}^{1 / 2-\alpha}-\nu_{1}^{1 / 2-\alpha}}{\nu_{2}^{1-\alpha}-\nu_{1}^{1-\alpha}} \frac{2-2 \alpha}{1-2 \alpha}
$$

Note, that the energy of relativistic particles $W_{p}$ in sources, when take into account the relativistic protons, may be

$$
W_{P}=100 \cdot W_{e}
$$

It is known, the value of the magnetic field energy $W_{B_{I C}}$ in sources is:

$$
W_{B_{I C}}=B_{I C}^{2} \cdot \frac{V}{8 \pi}
$$

where $V$ is a source volume.

Thus, we have estimated the energies of relativistic particles and magnetic field energies of objects and their ratio in the examined samples:

(sample $(\mathrm{S})$ ) for galaxies $\left\langle W_{p}\right\rangle=1.16( \pm 0.58) \cdot 10^{61} \mathrm{erg},\left\langle W_{B_{I C}}\right\rangle=1.71( \pm 1.65) \cdot 10^{62}$ $\mathrm{erg}, \quad\left\langle\frac{W_{p}}{W_{B_{I C}}}>=53.01( \pm 15.4)\right.$; for quasars $\left\langle W_{p}>=3.1( \pm 0.97) \cdot 10^{61} \mathrm{erg}, \quad\left\langle W_{B_{I C}}>=\right.\right.$ $3.12( \pm 1.37) \cdot 10^{62} \mathrm{erg},<\frac{W_{p}}{W_{B_{I C}}}>=3.12( \pm 1.53)$;

(sample $(\mathrm{C}+))$ for galaxies $\left\langle W_{p}\right\rangle=3.82( \pm 1.30) \cdot 10^{60} \mathrm{erg},\left\langle W_{B_{I C}}\right\rangle=1.97( \pm 1.64) \cdot 10^{60}$ $\mathrm{erg},\left\langle\frac{W_{p}}{W_{B_{I C}}}>=20071.5( \pm 16620)\right.$; for quasars $\left\langle W_{p}>=2.31( \pm 1.66) \cdot 10^{62} \mathrm{erg},\left\langle W_{B_{I C}}>=\right.\right.$ $6.76( \pm 2.94) \cdot 10^{61} \mathrm{erg},<\frac{W_{p}}{W_{B_{I C}}}>=19.55( \pm 10.14)$.

In this way, the estimated ratios of energies for the steep-spectrum radio sources indicate on prevalence of the relativistic particle's energy over the magnetic field energy. Note, from studies the X-ray emission of radio galaxies (with low and great radio power) and quasars a number of authors (Isobe et al. 2002, Croston et al. 2005, Hardcastle et al. 2007, Mikhailova et al. 2010, Mingo et al. 2012) have concluded that these sources appear to be further from energy equipartition, and the particle's energy exceeds the magnetic field energy in sources.

We have considered relationships of the ratio of energies on the cosmological epoch, linear size, contribution of low-frequency emission, characteristic age for examined sources.It is interesting, that considered steep-spectrum galaxies and quasars display the similar corresponding relations. The obtained relationships reveal the decrease of relativistic particle's energy at increase of linear size of a source, and at increase of the contribution of low-frequency emission in steep-spectrum sources. Besides, as can be seen from Fig. 2, the energy ratio is smaller at larger ages of steep-spectrum sources. Also, two branches of source's evolution may exist, and at that the activity recurrence may be supposed in examined sources.

\section{Conclusions}

The values of the magnetic field strength of steep-spectrum sources determined at the IC-mechanism are, in average, to by one order less than the corresponding values of magnetic field strength derived at well-known condition of energy equipartition. 


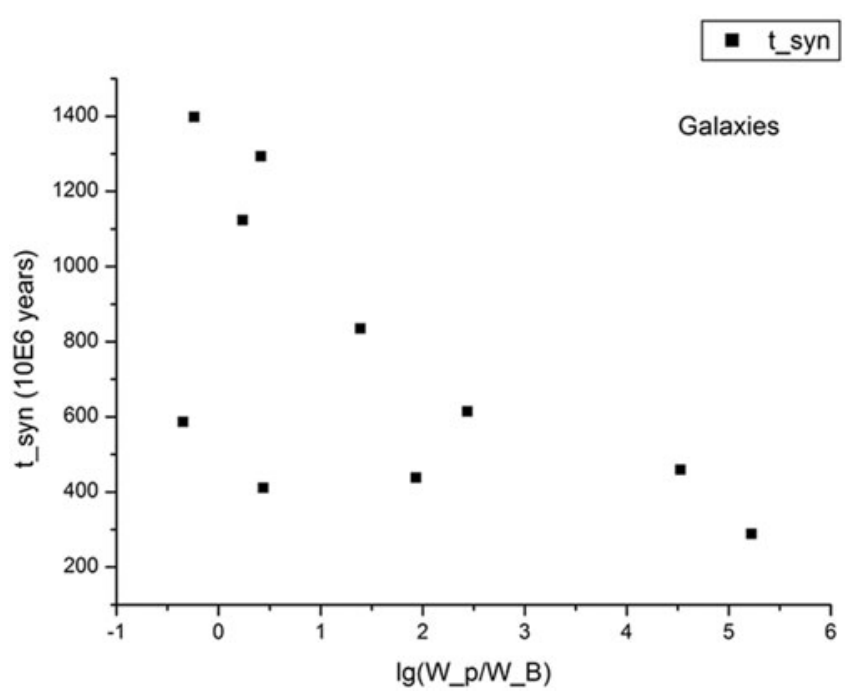

Figure 2. Characteristic age vs energy ratio for galaxies from sample $(\mathrm{C}+)$.

We conclude about the prevalence of the energy of relativistic particles over the energy of the magnetic field in the examined sources. The great extent of radio structure of quasars and galaxies with steep spectrum may evidence in favour of such situation.

The ratio of energies reveals relationships on the cosmological epoch, linear size, contribution of low-frequency emission, characteristic age of steep-spectrum sources. These may indicate on two branches of evolution of sources with steep radio spectra.

Also, these relationships may allow the possibility of activity recurrence of galaxies and quasars with steep radio spectra.

\section{References}

Braude, S. Y.a., Megn, A. V., Rashkovsky, S. L., et al. 1978, Astrophys. Space Sci., 54, 37

Braude, S. Y.a., Megn, A. V., Sokolov, K. P., et al. 1979, Astrophys. Space Sci., 64, 73

Braude, S. Y.a., Miroshnichenko, A. P., Sokolov, K. P., \& Sharykin, N. K. 1981a, Astrophys. Space Sci., 74, 409

Braude, S. Y.a., Miroshnichenko, A. P., Sokolov, K. P., \& Sharykin, N. K. 1981b, Astrophys. Space Sci., 76, 279

Braude, S. Y.a., Miroshnichenko, A. P., Rashkovsky, S. L., et al. 2003, Kin. Phys. Celest. Bodies, 19,291

Croston, J., Hardcastle, M., Harris, D., et al. 2005, ApJ, 626, 733

Hardcastle, M., Croston, J., \& Kraft, R. 2007, ApJ, 669, 893

Harris, D. \& Grindley, J. 1979, MNRAS, 188, 25

Isobe, N., Makishima, K., Iyomoto, N., et al. 2002, ApJ(Letters), 580, L111

Kardashev, N. S. 1962, Sv. Astron., 6, 317

Lang, K. 1974, Astrophysical Formulae (Berlin:Springer-Verlag)

Mikhailova, M. S., Bannikova, E. Y.u., \& Kontorovich, V. M. 2010, Astron.Reports, 54, 481

Mingo, B., Hardcastle, M., Croston, J., et al. 2012, ApJ, 758, 95

Miroshnichenko, A. P. 2012, Radio Physics and Radio Astronomy, 3, 215 